\title{
14. COCCOLITH STRATIGRAPHY, LEG 8, DEEP SEA DRILLING PROJECT ${ }^{1}$
}

\author{
David Bukry, U. S. Geological Survey, La Jolla, California
}

Leg 8 of the Deep Sea Drilling Project, OctoberDecember 1969, from Hawaii to Tahiti, recovered 181 cores at eight drilling sites (Figure 1). Light-microscope techniques were used to study the coccoliths of 297 samples from these cores. Following a summary of the coccolith stratigraphy at each drilling site, coccolith species identified in selected samples are listed by time-stratigraphic series and biostratigraphic zone. The coccolith zones used in this report are based on

${ }^{1}$ Publication authorized by the Director, U.S. Geological Survey. tentative zones described in the report on coccoliths from DSDP Leg 3 (Bukry and Bramlette, 1970), which were developed from the work of M. N. Bramlette and co-authors, and W. W. Hay and co-authors (see References). Recent improvements in Neogene zonation by Gartner (1969), which were briefly discussed in conjunction with the study of coccoliths from DSDP Leg 7 (Bukry, 1971), have been adopted. Zonal assignment of cores from Leg 8 holes is summarized in Table 3. The identified species are listed below in alphabetic order by genus name with authorship indicated and are then indexed in alphabetic order by trivial name.

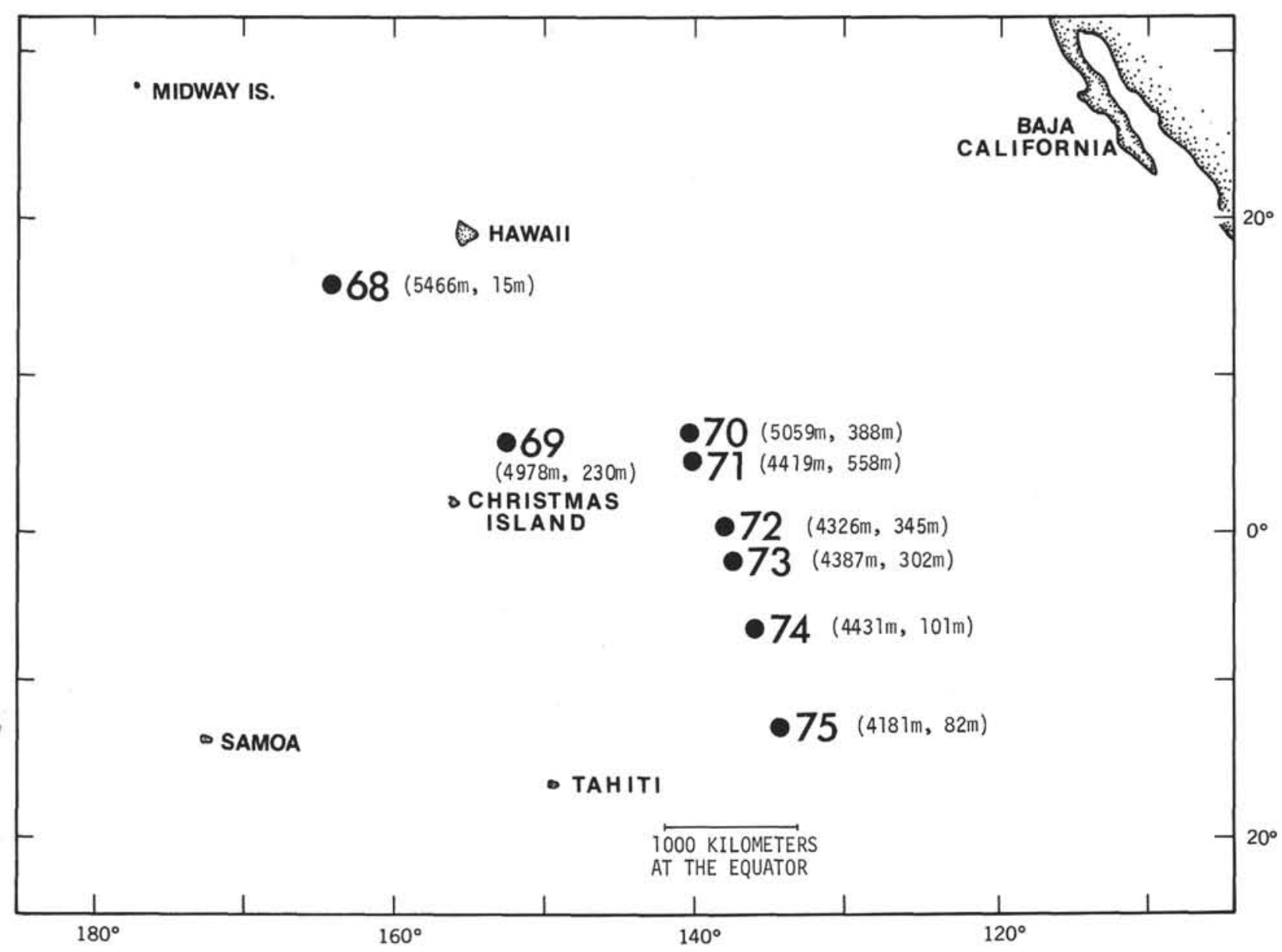

Figure 1. Location of drilling sites, Leg 8, Deep Sea Drilling Project. Site numbers are followed (in parentheses) by water depth and deepest drilling penetration. 
TABLE 1

Coccolith Species Considered

Bramletteius serraculoides Gartner

Campylosphaera dela (Bramlette and Sullivan)

Ceratolithus cristatus Kamptner

Ceratolithus rugosus Bukry and Bramlette

Ceratolithus tricorniculatus Gartner

Chiasmolithus grandis (Bramlette and Riedel)

Chiasmolithus oamaruensis (Deflandre)

Coccolithus bisectus (Hay, Mohler, and Wade) of Bramlette and Wilcoxon

Coccolithus doronicoides Black and Barnes

Coccolithus eopelagicus (Bramlette and Riedel)

Coccolithus fenestratus (Deflandre and Fert) basionym:

Discolithus fenestratus Deflandre and Fert, 1954, Ann.

Paleont. 40, p. 139, pl. 11, fig. 25; text-fig. 52.

Coccolithus pelagicus (Wallich)

Coccolithus stavensis Levin and Joerger

Coronocyclus serratus Hay, Mohler, and Wade

Cyclococcolithina formosa (Kamptner)

Cyclococcolithina leptopora (Murray and Blackman)

Cyclococcolithina macintyrei (Bukry and Bramlette)

Cyclococcolithina neogammation (Bramlette and Wilcoxon)

Discoaster adamanteus Bramlette and Wilcoxon

Discoaster asymmetricus Gartner

Discoaster barbadiensis Tan

Discoaster brouweri Tan

Discoaster brouweri rutellus Gärtner

Discoaster challengeri Bramlette and Riedel

Discoaster deflandrei Bramlette and Riedel

Discoaster dilatus Hay

Discoaster druggii Bramlette and Wilcoxon

Discoaster exilis Martini and Bramlette

Discoaster hamatus Martini and Bramlette

Discoaster kugleri Martini and Bramlette

Discoaster lidzi Hay

Discoaster neohamatus Bukry and Bramlette

Discoaster pentaradiatus Tan

Discoaster perplexus Bramlette and Riedel

Discoaster quinqueramus Gartner

Discoaster saipanensis Bramlette and Riedel

Discoaster surculus Martini and Bramlette

Discoaster tani nodifer Bramlette and Riedel
Discoaster tani tani Bramlette and Riedel

Discoaster variabilis Martini and Bramlette

Discoaster wemmelensis Achutan and Stradner

Emiliania annula (Cohen)

Emiliania huxleyi (Lohmann)

Gephyrocapsa oceanica Kamptner

Helicopontosphaera compacta (Bramlette and Wilcoxon)

Helicopontosphaera kamptneri Hay and Mohler

Helicopontosphaera parallela Bramlette and Wilcoxon Helicopontosphaera sellii Bukry and Bramlette

Isthmolithus recurvus Deflandre

Orthorhabdus serratus Bramlette and Wilcoxon

Pontosphaera vadosa Hay, Mohler, and Wade

Reticulofenestra gartneri (Roth and Hay)

Reticulofenestra pseudoumbilica (Gartner)

Reticulofenestra umbilica (Levin)

Sphenolithus abies Deflandre

Sphenolithus belemnos Bramlette and Wilcoxon Sphenolithus ciperoensis Bramlette and Wilcoxon

Sphenolithus distentus (Martini)

Sphenolithus furcatolithoides Locker

Sphenolithus heteromorphus Deflandre

Sphenolithus moriformis (Bronnimann and Stradner)

Sphenolithus neoabies Bukry and Bramlette

Sphenolithus predistentus Bramlette and Wilcoxon

Sphenolithus pseudoradians Bramlette and Wilcoxon

Triquetrorhabdulus carinatus Martini

Triquetrorhabdulus rugosus Bramlette and Wilcoxon

TABLE 2

Index of Coccolith Trivial Names Considered

abies, Sphenolithus

adamanteus, Discoaster

annula, Emiliania

asymmetricus, Discoaster

barbadiensis, Discoaster

belemnos, Sphenolithus

bisectus, Coccolithus

brouweri, Discoaster

brouweri rutellus, Discoaster

carinatus, Triquetrorhabdulus

challengeri, Discoaster 
ciperoensis, Sphenolithus

compacta, Helicopontosphaera

deflandrei, Discoaster

dela, Campylosphaera

dilatus, Discoaster

distentus, Sphenolithus

doronicoides, Coccolithus

druggii, Discoaster

eopelagicus, Coccolithus

exilis, Discoaster

fenestratus, Coccolithus

formosa, Cyclococcolithina

furcatolithoides, Sphenolithus

gartneri, Reticulofenestra

grandis, Chiasmolithus

hamatus, Discoaster

heteromorphus, Sphenolithus

huxleyi, Emiliania

kamptneri, Helicopontosphaera

kugleri, Discoaster

leptopora, Cyclococcolithina

lidzi, Discoaster

macintyrei, Cyclococcolithina

moriformis, Sphenolithus

neoabies, Sphenolithus

neogammation, Cyclococcolithina

neohamatus, Discoaster

oamaruensis, Chiasmolithus

oceanica, Gephyrocapsa

parallela, Helicopontosphaera

pelagicus, Coccolithus

pentaradiatus, Discoaster

perplexus, Discoaster

predistentus, Sphenolithus

pseudoradians, Sphenolithus

pseudoumbilica, Reticulofenestra

quinqueramus, Discoaster

recurvus, Isthmolithus

rugosus, Ceratolithus

rugosus, Triquetrorhabdulus

saipanensis, Discoaster

sellii, Helicopontosphaera

serraculoides, Bramletteius

\author{
serratus, Coronocyclus \\ serratus, Orthorhabdus \\ stavensis, Coccolithus \\ surculus, Discoaster \\ tani nodifer, Discoaster \\ tani tani, Discoaster \\ tricorniculatus, Ceratolithus \\ umbilica, Reticulofenestra \\ vadosa, Pontosphaera \\ variabilis, Discoaster \\ wermmelensis, Discoaster
}

Sample numbers given under the biostratigraphic zones for each site consist of elements in the following sequence: cruise-leg number; drill-hole designation, consisting of site number plus a letter, if more than one hole; core designation; core-section number; interval in centimeters below the top of each core section. For example, 8-69A-1A-6, $58-59 \mathrm{~cm}$, indicates that the sample came from Leg 8, Hole 69A (at Site 69), the first barrel of core recovered, the sixth section of that core and from 58 to 59 centimeters below the top of the section. Most core runs were 9.1 meters long, but occasionally the core liners were not full. In this report, the tops of recoveries are arbitrarily placed at the top of the core runs, and an approximate depth in meters below the sea floor follows each sample number.

\section{HOLES 68 AND 68A}

(lat $16^{\circ} 43.32^{\prime} \mathrm{N}$., long $164^{\circ} 10.36^{\prime} \mathrm{W}$., depth 4978 meters)

[No samples available; see report of shipboard scientists.]

\section{HOLES 69 AND 69A}

(lat $06^{\circ} 00.00^{\prime} \mathrm{N}$., long $152^{\circ} 51.93^{\prime} \mathrm{W}$., depth 4978 meters)

\section{Summary of Coccolith Stratigraphy}

Coccolith assemblages at this site range from middle Miocene at 15 meters to lower Oligocene at 143 meters. Most cores from the predominantly siliceous ooze encountered here contain few and poorly preserved coccoliths. Partial dissolution of heliolithid (radially constructed ring) coccoliths, such as Coccolithus eopelagicus, and of star-shaped discoasters, such as Discoaster brouweri s.l., is prevalent in Miocene sediment to a depth of 60 meters. Certain species generally typical for this interval-Reticulofenestra pseudoumbilica, Sphenolithus abies, and $S$. heteromorphus-are missing. Their absence makes difficult the assignment of Core 3 to either the Sphenolithus heteromorphus Zone or Discoaster exilis Zone, but 
TABLE 3

Series and Zone Assignment of Cores From Leg 8

\begin{tabular}{|c|c|c|c|c|c|c|c|c|c|c|c|}
\hline 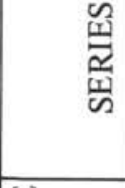 & Zone & 69 & $69 \mathrm{~A}$ & 70 & $70 \mathrm{~A}$ & 71 & 72 & $72 \mathrm{~A}$ & 73 & 74 & 75 \\
\hline \multirow{3}{*}{ 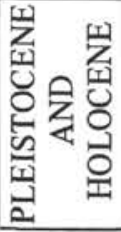 } & Emiliania huxleyi & & & & & \multirow{2}{*}{1} & & \multirow{2}{*}{1} & & \multirow{2}{*}{1} & \\
\hline & Gephyrocapsa oceanica & & & & & & 1 & & 1 & & \\
\hline & Coccolithus doronicoides & & & & & 1 & 1 & & $2-4$ & & \\
\hline \multirow{13}{*}{ 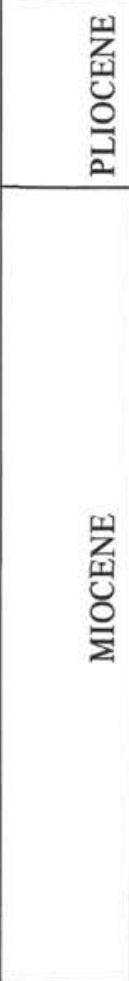 } & Discoaster brouweri & & & & & $1-2$ & & $2-4$ & $4-5$ & & \\
\hline & Reticulofenestra pseudoumbilica & & & & & 2 & & 5 & & & \\
\hline & Ceratolithus rugosus & & & & & 3 & 2 & 5 & $6-7,8$ & & \\
\hline & Ceratolithus tricorniculatus & & & & & 4 & $2-3$ & 6 & $7-8$ & & \\
\hline & Discoaster quinqueramus & & & & & \multirow{2}{*}{$5-7$} & \multirow{2}{*}{3} & & $8 ?$ & & \\
\hline & Discoaster neohamatus & & & & & & & & & & \\
\hline & Discoaster hamatus & & & & & 8 & & & & & \\
\hline & Catinaster coalitus & & & & & & & & & & \\
\hline & Discoaster exilis & $2-3$ & & 3 & & $9-15$ & 4 & & $8-9$ & & \\
\hline & Sphenolithus heteromorphus & & & 4 & & $16-24$ & 5 & & $10-11$ & & \\
\hline & Helicopontosphaera ampliaperta & & & & & 24 & & & & & \\
\hline & Sphenolithus belemnos & & & & & $25-26$ & & & & & \\
\hline & Triquetrorhabdulus carinatus & $3-4$ & & $6-12$ & $1-3$ & $28-40$ & 6 & & 12 & $3-5$ & $1-4$ \\
\hline \multirow{4}{*}{ 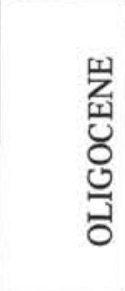 } & Sphenolithus ciperoensis & & $1-2$ & & 4-5 & $42-44$ & & & & $6-7$ & $4-5$ \\
\hline & Sphenolithus distentus & & $3-5$ & & $6-14$ & $46-48$ & $7-8$ & & 13 & & \\
\hline & Sphenolithus predistentus & 5 & 6 & & $14-25$ & & 8 & & $14-17$ & $8-10$ & $8-9$ \\
\hline & Helicopontosphaera reticulata & & 7.8 & & $25-27$ & & 9 & & 18 & 11 & \\
\hline \multirow{2}{*}{ 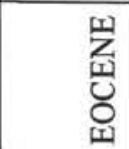 } & Discoaster barbadiensis & & & & & & $10-11$ & & $19-21$ & & \\
\hline & Reticulofenestra umbilica & & & & & & & & & 12 & \\
\hline
\end{tabular}


evidence provided by the abundance of Discoaster deflandrei among the discoasters indicates that the assemblage is lower middle Miocene. Ortholithid genera such as Triquetrorhabdulus and Discoaster seem to be the best preserved coccoliths in this siliceous ooze and are especially common in Core 4 . The lowest definitive coccolith assemblages representing the lower Oligocene Helicopontosphaera reticulata Zone are present in Cores 7A and 8A (129 to 144 meters). Samples from Cores 9A through 12A (151 meters to 224 meters) are siliceous ooze with only rare coccoliths that probably represent drilling contamination from higher Oligocene deposits.

\section{Coccoliths in Selected Samples, Hole 69 \\ Middle Miocene \\ (Discoaster exilis Zone)}

8-69-2-1, 63-65 cm; $15 \mathrm{~m}$ :

Coccolithus eopelagicus, Discoaster brouweri s.l., D. deflandrei, D. exilis, Triquetrorhabdulus rugosus.

\section{Middle Miocene \\ (Discoaster exilis Zone or Sphenolithus heteromorphus Zone)}

8-69-2-6, 63-65 cm; $22 \mathrm{~m}$ :

C. eopelagicus [centerless], Cyclococcolithina leptoporus, D. brouweri [centerless], D. deflandrei, $D$. exilis.

8-69-3-4, 63-64 cm; $28 \mathrm{~m}$ :

C. eopelagicus [centerless], Cyclococcolithina neogammation?, $D$. deflandrei, $D$. exilis.

\section{Lower Miocene}

(Triquetrorhabdulus carinatus Zone)

8-69-3-6, 63-64 cm; $31 \mathrm{~m}$ :

C. eopelagicus, C. pelagicus, C. neogammation [centerless], Discoaster sp. cf. D. deflandrei, Sphenolithus moriformis, Triquetrorhabdulus carinatus.

8-69-4-6, 63-64 cm; $60 \mathrm{~m}$ :

$C$. eopelagicus [centerless], $D$. sp. cf. $D$. deflandrei, $S$. moriformis, $T$. carinatus [abundant]

\section{Middle Oligocene}

(Sphenolithus predistentus Zone)

8-69-5-6, 63-64 cm; $125 \mathrm{~m}$ :

Coccolithus bisectus, C. eopelagicus, C. sp. cf. C. scissurus, $C$. neogammation, D. deflandrei, D. tani nodifer, D. tani tani, Sphenolithus sp. aff. S. belemnos, $S$. moriformis, $S$. predistentus.
Coccoliths in Selected Samples, Hole 69A

Upper Oligocene

(Sphenolithus ciperoensis Zone)

8-69A-1A-6, 58-59 cm; $69 \mathrm{~m}$ :

Coccolithus sp. aff. C. bisectus, C. eopelagicus, Cyclococcolithina neogammation, Discoaster sp. cf. D. deflandrei, Sphenolithus ciperoensis, S. sp. aff. S. belemnos, Triquetrorhabdulus carinatus.

8-69A-2A-6, 63-64 cm; $78 \mathrm{~m}:$

Coccolithus bisectus, $C$. sp. aff. C. bisectus, C. eopelagicus, C. pelagicus, C. neogammation, Discoaster deflandrei, $D$. sp. cf. D. tani nodifer, Reticulofenestra gartneri, $S$. ciperoensis, $S$. moriformis, $S$. sp. aff. $S$. belemnos, T. carinatus.

\section{Middle Oligocene \\ (upper Sphenolithus distentus Zone)}

8-69A-3A-1, 63-64 cm; $80 \mathrm{~m}$ :

$C$. sp. aff. C. bisectus, C. eopelagicus, $C$. pelagicus, $C$. neogammation, D. deflandrei, Sphenolithus distentus, $S$. moriformis, $S$. predistentus, $T$. carinatus.

8-69A-3A-3, 63-64 cm; $83 \mathrm{~m}$ :

C. bisectus, $C$. sp. aff. C. bisectus, C. eopelagicus, $C$. neogammation, $D$. deflandrei, $R$. gartneri, $S$. distentus, $S$. moriformis, $T$. carinatus.

8-69A-3A-5, $6-7 \mathrm{~cm} ; 85 \mathrm{~m}$ :

C. bisectus, $C$. sp. aff. C. bisectus, $C$. eopelagicus, $C$. pelagicus, $C$. neogammation, $D$. deflandrei, Sphenolithus sp. cf. S. ciperoensis, S. distentus, S. moriformis, T. carinatus.

\section{Middle Oligocene \\ (lower Sphenolithus distentus Zone)}

8-69A-4A-6, 63-64 cm; $97 \mathrm{~m}$ :

C. eopelagicus, C. pelagicus, C. neogammation, D. deflandrei, $S$. distentus, $S$. predistentus.

8-69A-5A-6, 63-64 cm; $106 \mathrm{~m}:$

C. bisectus, $C$. sp. aff. C. bisectus [rare], C. eopelagicus, $C$. neogammation, $D$. deflandrei, $D$. sp. cf. D. tani nodifer, D. tani tani [rare], $R$. gartneri, Sphenolithus sp. cf. S. ciperoensis, $S$. distentus, $S$. predistentus.

\section{Middle Oligocene \\ (Sphenolithus predistentus Zone)}

8-69A-6A-6, 63-64 cm; $115 \mathrm{~m}$ :

C. bisectus, C. pelagicus, C. neogammation, D. deflandrei, D. tani nodifer, D. tani tani, $R$. gartneri, $S$. predistentus, S. pseudoradians. 
Lower Oligocene

(Helicopontosphaera reticulata Zone)

8-69A-7A-3, 73-74 cm; 129 to $135 \mathrm{~m}$ :

C. bisectus, C. eopelagicus, C. pelagicus, Coccolithus stavensis, D. deflandrei, D. tani nodifer, Pontosphaera vadosa, R. gartneri, R. umbilica, S. moriformis [small], $S$. predistentus.

8-69A-8A-6, $63 \mathrm{~cm} ; 143 \mathrm{~m}$ :

Bramletteius serraculoides, $C$. bisectus, $C$. eopelagicus, Coccolithus stavensis, Cyclococcolithina formosus, $C$. neogammation, $D$. deflandrei, $D$. tani nodifer, $P$. vadosa, R. gartneri, R. umbilica, S. moriformis [small], $S$. predistentus.

8-69A-9A-5, 63-64 cm; $151 \mathrm{~m}$ :

8-69A-10A-6, $63 \mathrm{~cm} ; 161 \mathrm{~m}$ :

8-69A-11A-6, $63 \mathrm{~cm} ; 222 \mathrm{~m}$ :

8-69A-12A-1, $150 \mathrm{~cm} ; 224 \mathrm{~m}$ :

[Barren siliceous ooze or siliceous ooze with rare Oligocene contamination.]

\section{HOLES 70 AND 70A \\ (lat $06^{\circ} 20.08^{\prime} \mathrm{N}$., long $140^{\circ} 21.72^{\prime} \mathrm{W}$., depth 5059 meters)}

\section{Summary of Coccolith Stratigraphy}

As at Site 69, definitive coccolith assemblages are present for middle Miocene to lower Oligocene, and the upper cores consist of a siliceous-ooze facies in which coccolith assemblages show strong dissolution effects. A significant feature of this site is the expanded succession of well-preserved Oligocene coccolith assemblages from the Sphenolithus ciperoensis Zone at 148 meters to the Helicopontosphaera reticulata Zone at 322 meters.

\section{Coccoliths in Selected Samples, Hole 70}

\section{Middle Miocene}

(Discoaster exilis Zone, Discoaster kugleri Subzone)

8-70-3-3, 63-64 cm; $21 \mathrm{~m}$ :

Coccolithus eopelagicus, Cyclococcolithina macintyrei, Discoaster brouweri s.l., D. exilis, D. kugleri.

8-70-3-4, 63-64 cm; $22 \mathrm{~m}$ :

C. eopelagicus, Cyclococcolithina leptoporus, C. macintyrei, Discoaster dilatus, D. exilis, D. kugleri, Reticulofenestra pseudoumbilica.

\section{Middle Miocene (Discoaster exilis Zone, Coccolithus eopelagicus Subzone)}

8-70-3-5, $63-64 \mathrm{~cm} ; 24 \mathrm{~m}$ :

C. eopelagicus, C. leptoporus, $C$. macintyrei, Discoaster deflandrei, D. sp. cf. D. exilis, Helicopontosphaera kamptneri, R. pseudoumbilica, Triquetrorhabdulus rugosus.

\author{
Middle Miocene \\ (Sphenolithus heteromorphus Zone)
}

8-70-4-5, 63-64 cm; $33 \mathrm{~m}$ :

C. eopelagicus, Cyclococcolithina neogammation, $D$. brouweri s.l., D. deflandrei, D. exilis, D. sp. aff. $D$. variabilis, Sphenolithus heteromorphus.

\section{Lower Miocene}

(Triquetrorhabdulus carinatus Zone)

8-70-6-6, 63-64 cm; $52 \mathrm{~m}$ :

C. eopelagicus, $C$. pelagicus, $C$. neogammation, $D$. deflandrei, Sphenolithus sp. cf. $S$. belemnos, Triquetrorhabdulus carinatus.

8-70-8-6, 63-64 cm; $70 \mathrm{~m}$ :

Coccolithus sp. aff. C. bisectus, C. pelagicus, $C$. neogammation, $D$. deflandrei, $T$. carinatus.

8-70-10-6, 63-64 cm; $89 \mathrm{~m}$ :

$C$. eopelagicus, $C$. pelagicus, $C$. neogammation, $D$. deflandrei, $S$. sp. cf. $S$. belemnos, $S$. moriformis, $T$. carinatus [short].

8-70-11-6, 63-64 cm; $103 \mathrm{~m}$ :

$C$. eopelagicus, $C$. pelagicus, $C$. neogammation, $D$. deflandrei, D. druggii, S. moriformis, T. carinatus [short].

8-70-12-5, 63-64 cm; $111 \mathrm{~m}$ :

$C$. eopelagicus, $C$. pelagicus, $C$. neogammation, $D$. deflandrei, S. moriformis, $T$. carinatus.

Coccoliths in Selected Samples, Hole 70A

Lower Miocene

(Triquetrorhabdulus carinatus Zone)

8-70A-1A-6, 63-64 cm; $121 \mathrm{~m}$ :

$C$. pelagicus, $C$. neogammation, D. deflandrei, $S$. moriformis, $T$. carinatus.

\section{Lower Miocene}

(Triquetrorhabdulus carinatus Zone, Coccolithus sp. aff. C. bisectus Subzone)

8-70A-2A-6, 63-64 cm; $130 \mathrm{~m}$ :

$C$. sp. aff. $C$. bisectus, $C$. eopelagicus, $C$. pelagicus, $C$. neogammation, D. deflandrei, Reticulofenestra gartneri, Sphenolithus sp. aff. S. belemnos, S. moriformis, T. carinatus.

8-70A-3A-6, bottom; $140 \mathrm{~m}$ :

$C$. sp. aff. $C$. bisectus, $C$. eopelagicus, $C$. pelagicus, $C$. neogammation, D. deflandrei, Reticulofenestra gartneri, Sphenolithus sp. aff. S. belemnos, S. moriformis, T. carinatus.

8-70A-3A-6, bottom; $140 \mathrm{~m}$ :

$C$. sp. aff. C. bisectus, $C$. neogammation, $D$. deflandrei, $T$. carinatus. 


\section{Upper Oligocene \\ (Sphenolithus ciperoensis Zone)}

8-70A-4A-6, $63-64 \mathrm{~cm} ; 148 \mathrm{~m}$ :

Coccolithus bisectus [rare], $C$. sp. aff. C. bisectus, $C$. eopelagicus, $C$. pelagicus, $C$. neogammation, $D$. deflandrei, $S$. sp. aff. $S$. belemnos, $S$. ciperoensis [rare], $T$. carinatus.

8-70A-5A-6, $63-64 \mathrm{~cm} ; 157 \mathrm{~m}$ :

C. bisectus, C. sp. aff. C. bisectus, C. eopelagicus, $C$. pelagicus, $C$. neogammation, $D$. deflandrei, $S$. sp. aff. $S$. belemnos, S. ciperoensis, $S$. moriformis.

\section{Middle Oligocene \\ (Sphenolithus distentus Zone)}

8-70A-6A-6, 63-64 cm; $166 \mathrm{~m}$ :

$C$. sp. aff. C. bisectus, $C$. eopelagicus, $C$. pelagicus, $C$. neogammation, $D$. deflandrei, Sphenolithus distentus, $S$. moriformis [small], S. predistentus, T. carinatus [rare].

8-70A-7A-5, 63-64 cm; $175 \mathrm{~m}$ :

$C$. sp. aff. C. bisectus, C. eopelagicus, $C$. pelagicus, $C$. neogammation, $D$. deflandrei, Reticulofenestra gartneri, $S$. distentus, $S$. moriformis.

8-70A-8A-5, $63-64 \mathrm{~cm} ; 184 \mathrm{~m}$ :

Chiasmolithus sp. aff. $C$. oamaruensis, $C$. bisectus, $C$. sp. aff. $C$. bisectus, $C$. oepelagicus, $C$. pelagicus, $C$. sp. cf. C. scissurus, $C$. neogammation, $D$. deflandrei, $D$. sp. cf. D. tani tani [small], Helicopontosphaera sp. cf. $H$. parallela, S. distentus, S. moriformis.

8-70A-12A-4, $63-64 \mathrm{~cm} ; 215 \mathrm{~m}$ :

C. bisectus, C. sp. aff. C. bisectus, C. eopelagicus, $C$. pelagicus, $C$. neogammation, $D$. deflandrei, D. tani nodifer, R. gartneri, Sphenolithus sp. cf. S. ciperoensis, $S$. distentus.

Middle Oligocene

(Sphenolithus predistentus Zone)

8-70A-14A-3, $63-64 \mathrm{~cm} ; 232 \mathrm{~m}$ :

C. bisectus, C. sp. aff. C. bisectus, C. eopelagicus, $C$. pelagicus, $C$. neogammation, $D$. deflandrei, D. tani nodifer, $D$. tani tani, $S$. distentus, $S$. moriformis [small], S. predistentus.

8-70A-17A-4, $63-64 \mathrm{~cm} ; 261 \mathrm{~m}$ :

C. bisectus, C. eopelagicus, C. pelagicus, C. neogammation, D. deflandrei, D. tani nodifer, D. tani tani, Helicopontosphaera compacta, R. gartneri, S. moriformis, $S$. predistentus, $S$. pseudoradians.

8-70A-18A-2, $63-64 \mathrm{~cm} ; 264 \mathrm{~m}$ :

C. bisectus, C. eopelagicus, Coccolithus stavensis, $C$. neogammation, $D$. deflandrei, $D$. tani nodifer, $D$. tani tani, Reticulofenestra gartneri, $S$. predistentus, $S$. moriformis.
8-70A-19A-3, $63-64 \mathrm{~cm} ; 273 \mathrm{~m}$ :

$C$. bisectus, $C$. eopelagicus, $C$. pelagicus, $C$. stavensis, $C$. neogammation, $D$. deflandrei, $D$. tani tani, Pontosphaera vadosa, $R$. gartneri, $S$. moriformis, $S$. predistentus.

8-70A-23A-2, $64 \mathrm{~cm} ; 295 \mathrm{~m}$ :

C. bisectus, C. eopelagicus, $C$. pelagicus, $C$. stavensis, $C$. neogammation, $D$. deflandrei, $D$. tani nodifer, $D$. tani tani, R. gartneri, S. moriformis, S. predistentus.

8-70A-24A-1, 112-113 cm; $299 \mathrm{~m}:$

$C$. bisectus, $C$. eopelagicus, $C$. pelagicus, $C$. stavensis, $C$. neogammation, $D$. deflandrei, $D$. tani nodifer, $D$. tani tani, $H$. compacta, $S$. moriformis, $S$. predistentus.

8-70A-25A-2, $63 \mathrm{~cm} ; 306 \mathrm{~m}$ :

C. bisectus, $C$. eopelagicus, $C$. pelagicus, $C$. stavensis, $D$. deflandrei, D. tani nodifer, D. tani tani, P. vadosa, $R$. gartneri, S. moriformis [small form, abundant], $S$. predistentus.

\section{Lower Oligocene}

(upper Helicopontosphaera reticulata Zone)

8-70A-25A-3, $63-64 \mathrm{~cm} ; 308 \mathrm{~m}$ :

$C$. bisectus, $C$. eopelagicus, $C$. pelagicus, $C$. stavensis, $C$. neogammation, $D$. deflandrei, $D$. tani nodifer, $D$. tani tani, $H$. compacta, $P$. vadosa, R. gartneri, R. umbilica, $S$. moriformis, $S$. predistentus.

8-70A-26A-2, Bottom; $314 \mathrm{~m}$ :

Same as above.

Lower or Middle Oligocene (? Zone)

[Rare specimens in siliceous ooze]

8-70A-26A-3, 63-64 cm; $315 \mathrm{~m}$ :

C. bisectus, C. sp. aff. C. bisectus, C. pelagicus, $C$. stavensis, Cyclococcolithina formosus, C. neogammation, S. moriformis.

\section{Lower Oligocene \\ (Helicopontosphaera reticulata Zone)}

8-70A-27A-1, $175-176 \mathrm{~cm} ; 320 \mathrm{~m}$ :

Bramletteius serraculoides, Chiasmolithus oamaruensis, $C$. bisectus, $C$. eopelagicus, $C$. sp. cf. $C$. fenestratus, $C$. pelagicus, $C$. stavensis, Coronocyclus sp. cf. C. serratus, C. formosus, $D$. deflandrei, $D$. tani nodifer, D. tani tani, $H$. compacta, P. vadosa, $P$. umbilica, S. moriformis, $S$. predistentus.

8-70A-27A-2, $63-64 \mathrm{~cm} ; 322 \mathrm{~m}$ :

$C$. bisectus, $C$. eopelagicus, $C$. pelagicus, $C$. stavensis, $C$. formosus, $D$. deflandrei, $D$. tani tani, $P$. vadosa, $R$. umbilica. 


\section{HOLES 71 AND 71A \\ (lat $04^{\circ} 28.28^{\prime} \mathrm{N}$., long $140^{\circ} 18.91^{\prime} \mathrm{W}$., depth 4419 meters)}

\section{Summary of Coccolith Stratigraphy}

The 48 cores taken at Hole 71 provide an almost complete stratigraphic sequence from Holocene to middle Oligocene. The only missing assemblages are upper Miocene. In Cores 4 to 6 , which contain the upper Miocene Ceratolithus tricomiculatus Zone and Discoaster quinqueramus Zone or Discoaster neohamatus Zone, five-rayed specimens of Discoaster are abnormally uncommon. From 252 to 380 meters, the lowermost Miocene Triquetrorhabdulus carinatus Zone is well developed. Most of the specimens in assemblages of this zone from Site 71 show thick overgrowths of secondary calcite. The usual division of the zone into upper assemblages based on the presence of Discoaster druggii, Orthorhabdus serratus, and short forms of Triquetrorhabdulus carinatus and lower assemblages based on the abundance of Coccolithus sp. aff. $C$. bisectus and long forms of $T$. carinatus is applicable here, but the anomalous occurrence of a sample in Core 35 with common D. druggii is an exception. As in most mid-Tertiary tropical carbonate ooze, tiny and difficultly distinguished species of Sphenolithus are abundant and form a consistent background in most Oligocene samples examined from these cores.

Mid Pleistocene to Holocene (Gephyrocapsa oceanica Zone or Emiliania huxleyi Zone)

8-71-1-1, 63-64 cm; $1 \mathrm{~m}$ :

Ceratolithus cristatus, Cyclococcolithina leptoporus [small], Gephyrocapsa oceanica, Helicopontosphaera kamptneri, $H$. sellii, Syracosphaera sp.

Lower Pleistocene

(Coccolithus doronicoides Zone)

8-71-1-2, 63-64 cm; $2 \mathrm{~m}$ :

C. cristatus, Coccolithus sp. cf. C. doronicoides, $C$. leptoporus, H. kamptneri, Syracosphaera sp.

\section{Upper Pliocene \\ (Discoaster brouweri Zone, Cyclococcolithina macintyrei Subzone)}

8-71-1-3, 63-64 cm; $4 \mathrm{~m}$ :

Ceratolithus rugosus, $C$. sp. cf. C. doronicoides, $C$. pelagicus, C. leptoporus, Cyclococcolithus macintyrei, Discoaster brouweri, D. sp. aff. D. exilis.

\section{Upper Pliocene}

(Discoaster brouweri Zone, Discoaster pentaradiatus Subzone)

8-71-2-2, 25-26 cm; $11 \mathrm{~m}$ :

C. rugosus, C. leptoporus, C. macintyrei, D. brouweri, D. pentaradiatus, D. sp. cf. D. variabilis.

\section{Lower Pliocene \\ (Reticulofenestra pseudoumbilica Zone, Discoaster asymmetricus Subzone)}

8-71-2-3, 63-64 cm; $13 \mathrm{~m}$ :

$C$. rugosus, C. pelagicus, C. leptoporus, C. macintyrei, Discoaster asymmetricus, $D$. brouweri, D. exilis, $D$. pentaradiatus, D. variabilis, Reticulofenestra pseudoumbilica.

\section{Lower Pliocene \\ (Reticulofenestra pseudoumbilica Zone, Sphenolithus neoabies Subzone)}

8-71-2-5, 63-64 cm; $16 \mathrm{~m}$ :

C. rugosus, C. macintyrei, Discoaster challengeri, $D$. exilis, D. pentaradiatus, D. surculus, D. variabilis, $R$. pseudoumbilica.

\section{Lower Pliocene or Upper Miocene (Ceratolithus rugosus Zone)}

8-71-3-1, 63-64 cm; $19 \mathrm{~m}$ :

C. rugosus, C. tricorniculatus, C. leptoporus, C. macintyrei, D. brouweri, D. challengeri, D. pentaradiatus [rare], D. quinqueramus ] rare], D. surculus, $R$. pseudoumbilica.

8-71-3-6, top; $51 \mathrm{~m}$ :

C. rugosus, C. tricorniculatus, C. leptoporus, $C$. macintyrei, $D$. brouweri, $D$. challengeri, $D$. pentaradiatus, $D$. surculus, $D$. variabilis, $R$. pseudoumbilica.

\section{Upper Miocene}

(Ceratolithus tricorniculatus Zone)

8-71-4-5, 63-64 cm; $34 \mathrm{~m}$ :

C. tricorniculatus, C. pelagicus, C. macintyrei, $D$. brouweri s.l., D. challengeri, D. exilis [abundant], $D$. surculus, Triquetrorhabdulus rugosus.

\section{Upper Miocene \\ (Discoaster quinqueramus Zone or Discoaster neohamatus Zone)}

8-71-5-4, $63 \mathrm{~cm} ; 39 \mathrm{~m}$ :

C. pelagicus, C. macintyrei, D. brouweri, D. challengeri, D. surculus, $T$. rugosus.

8-71-6-4, $63 \mathrm{~cm} ; 48 \mathrm{~m}$ :

C. pelagicus, C. leptoporus, C. macintyrei, D. asymmetricus [rare], D. brouweri, D. challengeri, D. surculus, Sphenolithus neoabies, T. rugosus.

\section{8-71-7-2, $63 \mathrm{~cm} ; 54 \mathrm{~m}$ :}

$C$. pelagicus, $C$. macintyrei, $D$. brouweri, D. challengeri, $D$. neohamatus, $D$. sp. [small, 5 tapering rays], $R$. pseudoumbilica, T. rugosus. 


\section{Upper Miocene \\ (Discoaster hamatus Zone)}

8-71-8-5, 63-64 cm; $68 \mathrm{~m}$ :

C. pelagicus, C. leptoporus, C. macintyrei, D. challengeri, $D$. hamatus, $D$. sp. undescribed [small, 5 tapered rays, no central knob], R. pseudoumbilica, $T$. rugosus.

\section{Middle Miocene \\ (Discoaster exilis Zone)}

8-71-9-5, $63 \mathrm{~cm} ; 77 \mathrm{~m}$ :

Coccolithus eopelagicus, $C$. macintyrei, $D$. sp. cf. $D$. challengeri, $D$. sp. cf. D. exilis, $R$. pseudoumbilica, Sphenolithus neoabies, $T$. rugosus.

\section{Middle Miocene}

(Discoaster exilis Zone, Discoaster kugleri Subzone)

8-71-10-2, $63 \mathrm{~cm} ; 81 \mathrm{~m}$ :

C. eopelagicus, C. pelagicus, C. macintyrei, D. brouweri s.l., D. sp. cf. D. exilis, D. kugleri, H. kamptneri, $R$. pseudoumbilica, S. neoabies, T. rugosus.

8-71-10-5, $63 \mathrm{~cm} ; 86 \mathrm{~m}$ :

$C$. eopelagicus, $C$. leptoporus, $C$. macintyrei, $D$. sp. cf. D. exilis, D. kugleri, H. kamptneri, R. pseudoumbilica, T. rugosus.

\section{Middle Miocene \\ (Discoaster exilis Zone, Coccolithus eopelagicus Subzone)}

8-71-10-6, $63 \mathrm{~cm} ; 87 \mathrm{~m}$ :

C. eopelagicus, C. pelagicus, C. macintyrei, Discoaster deflandrei, $D$. sp. cf. $D$. exilis, $R$. pseudoumbilica, $S$. neoabies, $T$. rugosus.

8-71-13-5, $64 \mathrm{~cm} ; 114 \mathrm{~m}$ :

C. eopelagicus, C. leptoporus, D. sp. cf. D. exilis, $H$. kamptneri, $R$. pseudoumbilica, Sphenolithus sp. cf. $S$. abies, $T$. rugosus.

\section{8-71-15-5, $63 \mathrm{~cm} ; 132 \mathrm{~m}$ :}

C. eopelagicus, C. pelagicus, C. leptoporus, C. macintyrei, D. sp. cf. D. exilis, $R$. pseudoumbilica.

\section{Middle Miocene \\ (Sphenolithus heteromorphus Zone)}

8-71-16-2, 93-94 cm; $136 \mathrm{~m}$ :

C. eopelagicus, C. pelagicus, Cyclococcolithina neogammation, D. deflandrei, D. sp. cf. D. exilis, Sphenolithus heteromorphus.

8-71-20-6, $63 \mathrm{~cm} ; 178 \mathrm{~m}:$

C. eopelagicus, C. neogammation, D. deflandrei, $D$. sp. cf. D. exilis, H. kamptneri, S. heteromorphus.
8-71-24-4, $63 \mathrm{~cm} ; 212 \mathrm{~m}$ :

$C$. eopelagicus, $C$. neogammation, $D$. deflandrei, $D$. dilatus, $D$. exilis, $S$. heteromorphus.

\section{Lower Miocene}

(Helicopontosphaera ampliaperta Zone)

8-71-24-6, $63 \mathrm{~cm} ; 215 \mathrm{~m}$ :

C. pelagicus, C. neogammation, D. deflandrei [abundant], S. heteromorphus [few], S. moriformis.

Lower Miocene

(Sphenolithus belemnos Zone?)

8-71-26-6, $63 \mathrm{~cm} ; 233 \mathrm{~m}$ :

C. eopelagicus, C. pelagicus, C. neogammation, D. deflandrei, Sphenolithus belemnos.

\section{Lower Miocene \\ (Triquetrorhabdulus carinatus Zone, Discoaster druggii Subzone)}

8-71-28-6, $63 \mathrm{~cm} ; 252 \mathrm{~m}$ :

Coccolithus sp. aff. C. bisectus, C. eopelagicus, C. pelagicus, C. neogammation, D. deflandrei, $D$. sp. cf. $D$. druggii, Orthorhabdus serratus, Reticulofenestra gartneri, Sphenolithus sp. aff. S. belemnos.

\section{8-71-30-2, $63 \mathrm{~cm} ; 264 \mathrm{~m}$ :}

C. eopelagicus, C. pelagicus, C. neogammation, $D$. deflandrei, D. sp. cf. D. druggii, S. moriformis, Triquetrorhabdulus carinatus.

8-71-30-4, $63 \mathrm{~cm} ; 267 \mathrm{~m}:$

C. eopelagicus, C. pelagicus, C. neogammation, D. deflandrei, D. druggii, $D$. perplexus [fragmented], $S$. sp. aff. S. belemnos, $T$. carinatus.

\section{Lower Miocene}

(Triquetrorhabdulus carinatus Zone, Coccolithus sp. aff. C. bisectus Subzone)

8-71-30-6, $63 \mathrm{~cm} ; 270 \mathrm{~m}$ :

C. sp. aff. C. bisectus, C. eopelagicus, C. neogammation, D. deflandrei, T. carinatus.

8-71-32-6, $63 \mathrm{~cm} ; 288 \mathrm{~m}$ :

$C$. sp. aff. C. bisectus, $C$. eopelagicus, $C$. neogammation, D. deflandrei, $R$. gartneri, $S$. sp. aff. S. belemnos, T. carinatus.

8-71-33-6, $58 \mathrm{~cm} ; 298 \mathrm{~m}$ :

C. sp. aff. C. bisectus, C. pelagicus, C. neogammation, D. deflandrei, D. perplexus, Helicopontosphaera parallela, S. sp. aff. S. belemnos, S. moriformis, $T$. carinatus.

8-71-34-6, $63 \mathrm{~cm} ; 307 \mathrm{~m}$ :

C. eopelagicus, C. pelagicus, C. neogammation, D. deflandrei, $H$. parallela, S. belemnos [rare], $S$. sp. aff. $S$. belemnos. 


\section{Lower Miocene \\ (Triquetrorhabdulus carinatus Zone, Discoaster druggii Subzone)}

8-71-35-6, $63 \mathrm{~cm} ; 316 \mathrm{~m}$ :

C. neogammation, D. deflandrei, D. druggii [common], T. carinatus.

\section{Lower Miocene}

(Triquetrorhabdulus carinatus Zone, Coccolithus sp. aff. C. bisectus Subzone)

8-71-36-6, $63 \mathrm{~cm} ; 325 \mathrm{~m}$ :

$C$. sp. aff. C. bisectus, $C$. eopelagicus, $C$. pelagicus, $C$. neogammation, D. deflandrei, $T$. carinatus [abundant] .

8-71-38-6, $63 \mathrm{~cm} ; 344 \mathrm{~m}$ :

$C$. sp. aff. $C$. bisectus, $C$. neogammation, $D$. deflandrei,

$S$. sp. aff. $S$. belemnos, $S$. moriformis, $T$. carinatus.

8-71-40-5, $63 \mathrm{~cm} ; 361 \mathrm{~m}$ :

C. sp. aff. C. bisectus, C. pelagicus, C. neogammation,

$D$. deflandrei, $S$. sp. aff. $S$. belemnos, $S$. moriformis.

\section{Upper Oligocene \\ (Sphenolithus ciperoensis Zone)}

8-71-42-6, $63 \mathrm{~cm} ; 380 \mathrm{~m}$ :

$C$. sp. aff. $C$. bisectus, $C$. eopelagicus, $C$. pelagicus, $C$. neogammation, $D$. deflandrei, $R$. gartneri, $S$. sp. aff. $S$. belemnos, $S$. ciperoensis [rare], S. moriformis, $T$. carinatus.

8-71-44-6, $63 \mathrm{~cm} ; 398 \mathrm{~m}$ :

Coccolithus bisectus, $C$. sp. aff. $C$. bisectus, $C$. pelagicus, Coccolithus stavensis, C. neogammation, R. gartneri, S. ciperoensis [rare], S. moriformis [large and small varieties], $T$. carinatus.

\section{Middle Oligocene \\ (Sphenolithus distentus Zone)}

8-71-46-6, $63 \mathrm{~cm} ; 416 \mathrm{~m}$ :

C. bisectus, C. sp. aff. C. bisectus, C. eopelagicus, $C$. pelagicus, $C$. neogammation, $D$. deflandrei, Sphenolithus distentus, $S$. moriformis, $S$. predistentus, $T$. carinatus.

8-71-48-3, $63 \mathrm{~cm} ; 429 \mathrm{~m}$ :

$C$. bisectus, $C$. sp. aff. C. bisectus, C. pelagicus, $D$. neogammation, $D$. deflandrei, $R$. gartneri, $S$. distentus, $S$. moriformis [small], S. predistentus.

\section{Coccoliths in Selected Samples, Hole 71A}

[No samples available. See report of shipboard scientists].

\author{
HOLES 72 AND 72A \\ (lat $00^{\circ} 26.49^{\prime} \mathrm{N}$., long $138^{\circ} 52.02^{\prime} \mathrm{W}$., \\ depth 4326 meters)
}

\section{Summary of Coccolith Stratigraphy}

Cores from the surface to 344 meters recovered coccolith assemblages from Holocene to upper Eocene. Below the upper Miocene at 114 meters, coring gaps are present and several zones are not represented (Table 3). Several unusual species are present in Miocene and Pliocene sediment that contains common diatoms and radiolarians. Near the Miocene-Pliocene boundary in Cores 2 and 5A several types of Ceratolithus transitional between $C$. rugosus and $C$. tricorniculatus are present. Within the upper Miocene Core 3, a gigantic discoaster similar to Discoaster brouweri occurs that has been observed in upper Miocene samples from other Deep Sea Drilling Project sites. In the middle Miocene Core 4, other noteworthy coccoliths include a variety of Triquetrorhabdulus rugosus that shows refringence in polarized light and a variety of Sphenolithus abies that has very elongate spines, giving the appearance of a pincushion.

\section{Coccoliths in Selected Samples, Hole 72}

\section{Pleistocene \\ (Gephyrocapsa oceanica Zone)}

8-72-1-1, $63 \mathrm{~cm} ; 1 \mathrm{~m}$ :

Coccolithus sp. cf. C. doronicoides, Cyclococcolithina leptoporus, Gephyrocapsa oceanica, Helicopontosphaera kamptneri, $H$. sellii.

\section{Pleistocene \\ (Coccolithus doronicoides Zone)}

8-72-1-2, $63 \mathrm{~cm} ; 2 \mathrm{~m}$ :

Ceratolithus cristatus, Coccolithus doronicoides, C. leptoporus, Emiliania annula, H. kamptneri.

8-72-1-6, $63 \mathrm{~cm} ; 8 \mathrm{~m}:$

C. cristatus, C. doronicoides, C. leptoporus, Gephyrocapsa sp., H. kamptneri.

\section{Upper Miocene or Lower Pliocene (Ceratolithus rugosus Zone)}

8-72-2-1, $126 \mathrm{~cm} ; 61 \mathrm{~m}$ :

Ceratolithus sp. cf. C. rugosus, C. tricorniculatus, Coccolithus pelagicus, C. leptoporus, C. macintyrei, Discoaster brouweri, D. surculus, Reticulofenestra pseudoumbilica.

\section{8-72-2-3, $63 \mathrm{~cm} ; 64 \mathrm{~m}:$}

C. sp. cf. C. rugosus, C. tricorniculatus, $C$. pelagicus, $C$. leptoporus, C. macintyrei, Discoaster asymmetricus, $D$. brouweri, D. surculus, $H$. sellii, $R$. pseudoumbilica. 


\section{Upper Miocene \\ (Ceratolithus tricorniculatus Zone)}

8-72-2-4, $63 \mathrm{~cm} ; 65 \mathrm{~m}$ :

C. tricorniculatus, C. leptoporus, C. macintyrei, Discoaster perplexus [rare], D. quinqueramus, D. surculus, $R$. pseudoumbilica, Triquetrorhabdulus rugosus.

8-72-2-6, $63 \mathrm{~cm} ; 68 \mathrm{~m}$ :

C. tricorniculatus, C. pelagicus, C. leptoporus, C. macintyrei, $D$. brouweri, $D$. exilis, $D$. pentaradiatus, $D$. quinqueramus, D. surculus, D. variabilis, $R$. pseudoumbilica, Sphenolithus neoabies, T. rugosus.

8-72-3-2, $63 \mathrm{~cm} ; 108 \mathrm{~m}$ :

C. tricorniculatus, C. pelagicus, C. leptoporus, C. macintyrei, $D$. brouweri, $D$. challengeri, $D$. exilis, $D$. surculus, D. variabilis, $T$. rugosus.

8-72-3-3, $63 \mathrm{~cm} ; 110 \mathrm{~m}$ :

C. tricorniculatus [early variety, rare], C. pelagicus, $C$. leptoporus, C. macintyrei, D. brouweri s.l., D. variabilis, Sphenolithus abies, T. rugosus.

\section{Upper Miocene \\ (Discoaster quinqueramus Zone or \\ Discoaster neohamatus Zone)}

8-72-3-4, $63 \mathrm{~cm} ; 111 \mathrm{~m}$ :

$C$. pelagicus, $C$. leptoporus, $C$. macintyrei, $D$. brouweri s.l. [some abnormally large], D. challengeri s.l., $H$. kamptneri, R. pseudoumbilica, S. abies, T. rugosus.

8-72-3-5, $63 \mathrm{~cm} ; 113 \mathrm{~m}$ :

8-72-3-6, $63 \mathrm{~cm} ; 114 \mathrm{~m}$ :

Same as above with a few Discoaster sp. cf. $D$. quinqueramus.

\section{Middle Miocene}

(Discoaster exilis Zone)

8-72-4-1, $63 \mathrm{~cm} ; 151 \mathrm{~m}$ :

Coccolithus eopelagicus, C. pelagicus, C. macintyrei, Discoaster deflandrei, $D$. sp. cf. D. exilis, $R$. pseudoumbilica, $T$. rugosus.

$8-72-4-5,63 \mathrm{~cm} ; 157 \mathrm{~m}$ :

C. eopelagicus, C. leptoporus, C. macintyrei, D. brouweri s.l., $D$. deflandrei, $D$. sp. cf. $D$. druggii, $D$. sp. cf. $D$. exilis, $R$. pseudoumbilica, T. rugosus [many with slight refringence].

Middle Miocene

(Sphenolithus heteromorphus Zone)

8-72-5-1, $63 \mathrm{~cm} ; 212 \mathrm{~m}$ :

C. eopelagicus, $C$. pelagicus, C. leptoporus, C. neogammation, D. brouweri s.l., D. deflandrei, D. sp. cf. $D$. exilis, $R$. pseudoumbilica, Sphenolithus heteromorphus.
Lower Miocene

(Triquetrorhabdulus carinatus Zone, Coccolithus sp. aff. C. bisectus Subzone)

8-72-6-2, $63 \mathrm{~cm} ; 268 \mathrm{~m}$ :

Coccolithus sp. aff. C. bisectus, C. pelagicus [abundant], C. neogammation, D. deflandrei, Sphenolithus belemnos, $S$. sp. aff. $S$. belemnos, Triquetrorhabdulus carinatus [abundant] .

8-72-6-6, $63 \mathrm{~cm} ; 274 \mathrm{~m}:$

$C$. sp. aff. $C$. bisectus, $C$. eopelagicus, $C$. pelagicus, $C$. neogammation, $D$. deflandrei, $S$. sp. aff. $S$. belemnos, $S$. moriformis, $T$. carinatus.

Middle Oligocene

(Sphenolithus distentus Zone)

8.72-7-6, $63 \mathrm{~cm} ; 320 \mathrm{~m}$ :

Coccolithus bisectus, $C$. sp. aff. C. bisectus, $C$. eopelagicus, C. pelagicus, C. neogammation, D. deflandrei, Reticulofenestra gartneri, Sphenolithus distentus, $S$. predistentus.

\section{Middle Oligocene (Sphenolithus distentus Zone or Sphenolithus predistentus Zone)}

8-72-8-6, $64 \mathrm{~cm} ; 329 \mathrm{~m}$ :

C. bisectus, C. sp. aff. C. bisectus, C. pelagicus, $C$. neogammation, $D$. deflandrei, $R$. gartneri, $S$. sp. cf. $S$. distentus, $S$. predistentus, $S$. pseudoradians.

\section{Lower Oligocene \\ (Helicopontosphaera reticulata Zone)}

8-72-9-2, $63 \mathrm{~cm} ; 332 \mathrm{~m}$ :

Bramletteius serraculoides, $C$. bisectus [abundant], $C$. eopelagicus, C. pelagicus, Cyclococcolithina formosus, $D$. deflandrei, D. tani tani, Helicopontosphaera compacta, Pontosphaera vadosa, $R$. gartneri, $R$. umbilica.

8-72-9-6, $63 \mathrm{~cm} ; 338 \mathrm{~m}$ :

$C$. bisectus, $C$. pelagicus, $C$. formosus, $D$. deflandrei, $D$. tani nodifer, D. tani tani, P. vadosa, R. umbilica.

\section{Upper Eocene \\ (Discoaster barbadiensis Zone)}

8-72-10-3, 100-110 $\mathrm{cm} ; 339$ to $342 \mathrm{~m}$ :

B. serraculoides, C. bisectus, C. formosus, Discoaster barbadiensis, $D$. deflandrei, $D$. saipanensis, $R$. umbilica. Miocene or Pliocene taxa present as drilling contaminants: $C$. leptoporus, $D$. brouweri, $D$. exilis, $D$. variabilis.

8-72-10-5, 120-135 $\mathrm{cm} ; 339$ to $342 \mathrm{~m}$ :

$C$. bisectus, $C$. eopelagicus, $C$. formosus, $D$. barbadiensis, $D$. deflandrei, D. saipanensis, $R$. umbilica. 
8-72-11-1, 42-43 cm; $344 \mathrm{~m}$ :

$C$. bisectus, $C$. eopelagicus, $C$. formosus, $D$. barbadiensis, $D$. deflandrei, $D$. saipanensis [abundant], $D$. tani nodifer, $R$. umbilica.

Coccoliths in Selected Samples, Hole 72A

$$
\begin{gathered}
\text { Holocene or Pleistocene } \\
\text { (Emiliania huxleyi Zone or } \\
\text { Gephyrocapsa oceanica Zone) }
\end{gathered}
$$

8-72A-1A-1, $110 \mathrm{~cm} ; 9 \mathrm{~m}$ :

Cyclococcolithina leptoporus, Helicopontosphaera kamptneri, [a myriad of tiny coccoliths, possibly Emiliania huxleyi].

8-72A-1A-4, $63 \mathrm{~cm} ; 13 \mathrm{~m}$ :

Ceratolithus cristatus, C. leptoporus, Gephyrocapsa oceanica, H. kamptneri, $H$. sellii.

\section{Upper Pliocene \\ (Discoaster brouweri Zone, \\ Cyclococcolithina macintyrei Subzone)}

8-72A-2A-5, $63 \mathrm{~cm} ; 25 \mathrm{~m}$ :

Ceratolithus rugosus, Coccolithus pelagicus, C. leptoporus, C. macintyrei, Discoaster brouweri, H. kamptneri.

8-72A-3A-4, $63 \mathrm{~cm} ; 31 \mathrm{~m}$ :

C. rugosus, C. pelagicus, C. leptoporus, C. macintyrei,

D. brouweri, $H$. kamptneri, $H$. sellii.

\section{Upper Pliocene \\ (Discoaster brouweri Zone, Discoaster pentaradiatus Subzone)}

8-72A-3A-5, $63 \mathrm{~cm} ; 33 \mathrm{~m}$ :

C. rugosus, C. pelagicus, C. macintyrei, Discoaster asymmetricus, $D$. brouweri, $D$. pentaradiatus, $D$. surculus.

8-72A-4A-3, $63 \mathrm{~cm} ; 40 \mathrm{~m}$ :

$C$. rugosus, $C$. pelagicus, $C$. macintyrei, $D$. asymmetricus [common], D. brouweri, $D$. pentaradiatus, $D$. surculus, $D$. sp. cf. D. variabilis.

Lower Pliocene

(Reticulofenestra pseudoumbilica Zone)

8-72A-5A-2, $63 \mathrm{~cm} ; 47 \mathrm{~m}$ :

C. rugosus, C. leptoporus, C. macintyrei, D. brouweri, D. pentaradiatus, D. variabilis, Reticulofenestra pseudoumbilica, Sphenolithus neoabies.

\section{Upper Miocene or Lower Pliocene} (Ceratolithus rugosus Zone)

\section{8-72A-5A-4, $63 \mathrm{~cm} ; 50 \mathrm{~m}$ :}

$C$. rugosus, $C$. sp. aff. C. tricorniculatus, C. leptoporus, $C$. macintyrei, $D$. asymmetricus [rare], $D$. brouweri, $D$. exilis, $D$. pentaradiatus, $D$. surculus, $D$. variabilis, $R$. pseudoumbilica, Sphenolithus abies, S. neoabies.
8-72A-5A-6, $63 \mathrm{~cm} ; 53 \mathrm{~m}$ :

C. rugosus, C. tricorniculatus, $C$. pelagicus, $C$. leptoporus, $C$. macintyrei, $D$. brouweri, $D$. pentaradiatus [rare], D. surculus, $R$. pseudoumbilica.

\section{Upper Miocene \\ (Ceratolithus tricorniculatus Zone)}

8-72A-6A-6, $63 \mathrm{~cm} ; 62 \mathrm{~m}$ :

C. tricorniculatus, C. pelagicus, C. leptoporus, C. macintyrei, $D$. asymmetricus [rare], $D$. brouweri, $D$. challengeri, $D$. exilis, $D$. pentaradiatus [rare], $D$. surculus, D. variabilis, H. kamptneri [rare], R. pseudoumbilica, Triquetrorhabdulus rugosus [rare] .

\section{HOLE 73 \\ (lat $01^{\circ} 54.58^{\prime}$ S., long $137^{\circ} 28.12^{\prime} \mathrm{W}$., depth 4387 meters)}

\section{Summary of Coccolith Stratigraphy}

Upper Pleistocene assemblages are present in Core 1 at 2 meters, and Tertiary assemblages in the Pliocene, upper upper Miocene, middle Miocene, and Oligocene are well developed at this site as at Sites 71 and 72 . The hole terminates at 302 meters in upper Eocene radiolarian-rich sediment. Ceratolithus specimens transitional between $C$. rugosus and $C$. tricormiculatus occur in cores with Miocene-Pliocene boundary assemblages. A re-occurrence of Ceratolithus rugosus Zone assemblages at the top of Core 7 is probably the result of inhole slumping, as the older Ceratolithus tricorniculatus Zone assemblage is present at the bottom of the overlying Core 6. An unconformity is probably represented in Core 8, as an upper upper Miocene Discoaster quinqueramus Zone to Ceratolithus tricorniculatus Zone assemblage occurs at 63 meters and an upper middle Miocene assemblage of the Discoaster kugleri Subzone occurs at 66 meters.

Coccoliths in Selected Samples, Hole 73

Pleistocene

(Gephyrocapsa oceanica Zone)

8-73-1-2, $63 \mathrm{~m} ; 2 \mathrm{~m}$ :

Ceratolithus cristatus, Cyclococcolithina leptoporus, Gephyrocapsa oceanica.

\section{Pleistocene \\ (Coccolithus doronicoides Zone)}

8-73-2-5, $63 \mathrm{~cm} ; 8 \mathrm{~m}$ :

C. cristatus, Coccolithus doronicoides, C. leptoporus, C. macintyrei, Emiliania annula.

8-73-4-1, $63 \mathrm{~cm} ; 22 \mathrm{~m}$ :

C. cristatus, C. doronicoides, C. pelagicus, C. leptoporus, C. macintyrei, E. annula, Helicopontosphaera sellii. 


\section{Upper Pliocene \\ (Discoaster brouweri Zone, \\ Cyclococcolithina macintyrei Subzone)}

8-73-4-2, $63 \mathrm{~cm} ; 23 \mathrm{~m}$ :

Ceratolithus rugosus, C. pelagicus, C. leptoporus, $C$. macintyrei, Discoaster brouweri [rare] .

8-73-4-3, $63 \mathrm{~cm} ; 25 \mathrm{~m}$ :

C. rugosus, C. pelagicus, C. leptoporus, C. macintyrei, D. brouweri [abundant], Helicopontosphaera kamptneri.

\section{Upper Miocene or Lower Pliocene (Ceratolithus rugosus Zone)}

8-73-6-6, 63-64 cm; $48 \mathrm{~m}$ :

Ceratolithus sp. cf. C. rugosus, C. tricorniculatus, $C$. macintyrei, $D$. brouweri, $D$. exilis, $D$. surculus, $R$. pseudoumbilica.

8-73-7-1, 63-64 cm; $50 \mathrm{~m}$ :

C. rugosus, Ceratolithus sp. aff. C. tricorniculatus, $C$. pelagicus, $C$. macintyrei, Discoaster asymmetricus, $D$. brouweri, $D$. exilis, D. surculus, D. variabilis, $H$. kamptneri, $R$. pseudoumbilica.

8-73-7-3, $63-64 \mathrm{~cm} ; 53 \mathrm{~m}$ :

C. rugosus, C. leptoporus, C. macintyrei, D. brouweri, $D$. deflandrei, $D$. exilis, $D$. surculus, $R$. pseudoumbilica.

8-73-7-4, 63-64 cm; $54 \mathrm{~m}$ :

$C$. rugosus, $C$. sp. aff. C. tricorniculatus, $C$. pelagicus, C. leptoporus, C. macintyrei, D. asymmetricus, D. brouweri, $D$. pentaradiatus, $H$. sellii, $R$. pseudoumbilica.

8-73-7-5, 63-64 cm; $56 \mathrm{~m}$ :

$C$. sp. cf. C. rugosus, C. tricorniculatus, C. pelagicus, $C$. leptoporus, C. macintyrei, D. brouweri, D. deflandrei, $D$. exilis, $D$. surculus, $D$. variabilis.

\section{Upper Miocene \\ (Ceratolithus tricorniculatus Zone)}

8-73-7-6, 63-64 cm; $57 \mathrm{~m}$ :

C. tricorniculatus, C. leptoporus, C. macintyrei, D. brouweri rutellus, $D$. exilis, $D$. quinqueramus, $D$. surculus, D. variabilis, R. pseudoumbilica [small], Triquetrorhabdulus rugosus.

Upper Miocene or Lower Pliocene (Ceratolithus rugosus Zone) [Slumped downhole?]

8-73-8-1, 63-64 cm; $59 \mathrm{~m}$ :

C. sp. cf. C. rugosus, C. tricorniculatus, $C$. sp. aff. $C$. tricorniculatus, C. pelagicus, C. leptoporus, C. macintyrei, $D$. asymmetricus, $D$. brouweri, $D$. deflandrei, $D$. exilis, D. surculus, R. pseudoumbilica.
8-73-8-3, 6-7 cm; $61 \mathrm{~m}$ :

C. tricorniculatus, C. sp. aff. C. tricorniculatus, C. pelagicus, C. leptoporus, D. asymmetricus [rare], $D$. brouweri, $D$. challengeri, $D$. exilis, $D$. surculus, $R$. pseudoumbilica, T. rugosus [rare].

\section{Upper Miocene \\ (Ceratolithus tricorniculatus Zone)}

8-73-8-3, 63-64 cm; $62 \mathrm{~m}$ :

C. tricorniculatus, C. leptoporus, D. brouweri [large],

$D$. deflandrei, $D$. exilis, D. quinqueramus, $D$. surculus,

$R$. pseudoumbilica, $T$. rugosus [rare] .

\section{Upper Miocene \\ (Discoaster quinqueramus Zone or Ceratolithus tricorniculatus Zone)}

8-73-8-4, 63-64 cm; $63 \mathrm{~m}$ :

$C$. pelagicus, $C$. leptoporus, C. macintyrei, D. brouweri s.l., D. challengeri, D. deflandrei, D. exilis, D. surculus, $D$. variabilis, R. pseudoumbilica [rare], T. rugosus.

\section{Middle Miocene \\ (Discoaster exilis Zone, Discoaster kugleri Subzone)}

8-73-8-6, $63 \mathrm{~cm} ; 66 \mathrm{~m}$ :

Coccolithus eopelagicus, C. leptoporus, C. macintyrei, $D$. brouweri s.l., D. deflandrei, D. exilis [abundant, with a few five-rayed specimens], $D$. kugleri, $D$. variabilis [abundant], $R$. pseudoumbilica, T. rugosus.

8-73-9.6, $63 \mathrm{~cm} ; 75 \mathrm{~m}$ :

C. eopelagicus, C. pelagicus, C. leptoporus, C. macintyrei, D. kugleri, D. exilis, R. pseudoumbilica, $T$. rugosus.

\section{Middle Miocene}

(Sphenolithus heteromorphus Zone)

8-73-10-6, $63 \mathrm{~cm} ; 84 \mathrm{~m}$ :

C. eopelagicus, $C$. pelagicus, $C$. leptoporus, $C$. neogammation, D. deflandrei, D. sp. cf. D. exilis, Sphenolithus heteromorphus.

8-73-11-6, $63 \mathrm{~cm} ; 93 \mathrm{~m}:$

C. eopelagicus, C. pelagicus, C. leptoporus, C. neogammation, $D$. deflandrei, $D$. exilis, $S$. heteromorphus.

\section{Lower Miocene \\ (Triquetrorhabdulus carinatus Zone)}

8-73-12-2, $63 \mathrm{~cm} ; 142 \mathrm{~m}$ :

C. eopelagicus, C. pelagicus, C. neogammation, D. deflandrei, Sphenolithus sp. aff. S. belemnos, S. moriformis, Triquetrorhabdulus carinatus. 
Lower Miocene

(Triquetrorhabdulus carinatus Zone, Coccolithus sp. aff. C. bisectus Subzone)

8-73-12-6, $63 \mathrm{~cm} ; 148 \mathrm{~m}$ :

Coccolithus sp. aff. C. bisectus, C. pelagicus, $C$. neogammation, $D$. deflandrei, Reticulofenestra gartneri, $S$. sp. aff. $S$. belemnos, $S$. moriformis, $T$. carinatus.

\section{Middle Oligocene \\ (Sphenolithus distentus Zone)}

8-73-13-2, $63 \mathrm{~cm} ; 208 \mathrm{~m}$ :

Coccolithus bisectus, $C$. sp. aff. C. bisectus, C. eopelagicus, $C$. pelagicus, C. neogammation, $D$. deflandrei, $R$. gartneri, Sphenolithus distentus, S. pseudoradians.

Middle Oligocene

(Sphenolithus predistentus Zone)

8-73-14-2, $63 \mathrm{~cm} ; 245 \mathrm{~m}$ :

C. bisectus, $C$. eopelagicus, $C$. pelagicus, $C$. neogammation, $D$. deflandrei, D. tani nodifer, D. tani tani, Pontosphaera vadosa, $S$. moriformis, $S$. predistentus.

8-73-17-6, $63 \mathrm{~cm} ; 278 \mathrm{~m}:$

C. bisectus, C. pelagicus, $C$. neogammation, $D$. deflandrei, D. tani tani, Helicopontosphaera compacta, $R$. gartneri, $R$. umbilica [rare], S. predistentus.

\section{Lower Oligocene \\ (Helicopontosphaera reticulata Zone)}

8-73-18-6, $63 \mathrm{~cm} ; 287 \mathrm{~m}$ :

Bramletteius serraculoides [rare], C. bisectus, C. pelagicus, Cyclococcolithina formosus, C. neogammation, D. deflandrei, D. tani tani, $R$. umbilica.

\section{Upper Eocene \\ (Discoaster barbadiensis Zone)}

8-73-19-2, $63 \mathrm{~cm} ; 290 \mathrm{~m}$ :

$C$. bisectus, C. eopelagicus, $C$. pelagicus, $C$. formosus, Discoaster barbadiensis, $D$. saipanensis, $D$. tani nodifer, R. umbilica.

8-73-19-4, $63 \mathrm{~cm} ; 293 \mathrm{~m}:$

$B$. serraculoides, C. eopelagicus, C. pelagicus, C. formosus, D. barbadiensis, D. saipanensis, D. tani tani, $R$. umbilica.

8-73-19-6, $63 \mathrm{~cm} ; 296 \mathrm{~m}$ :

C. bisectus, C. eopelagicus, C. pelagicus, C. formosus, $D$. barbadiensis, $D$. saipanensis, $D$. tani tani, $R$. umbilica, Sphenolithus sp. cf. S. predistentus.

$8-73-21-2,63 \mathrm{~cm} ; 300 \mathrm{~m}$ :

$C$. bisectus, $C$. eopelagicus, $C$. pelagicus, $D$. barbadiensis, D. saipanensis, $H$. compacta, $R$. umbilica. Taxa representing Pliocene contamination: $C$. rugosus, $C$. macintyrei, $D$. brouweri.

\section{HOLE 74 \\ (lat $06^{\circ} 14.20^{\prime} \mathrm{S}$., long $136^{\circ} 05.80^{\prime} \mathrm{W}$., depth 4431 meters)}

\section{Summary of Coccolith Stratigraphy}

Sparse Pleistocene to Holocene coccoliths are present in the siliceous ooze of Core 1 at 2 meters, but basal Miocene sediment with abundant coccoliths is present in Core 3 at 21 meters [no intervening samples available], suggesting a greater hiatus than is present at Site 73. Oligocene sediment is present in Cores 6 through 11 ; and Core 12 at 102 meters is middle Eocene, the occurrence of centerless coccoliths indicating partial dissolution of calcite from this oldest sediment.

\section{Coccoliths in Selected Samples, Hole 74 \\ Pleistocene or Holocene (? Zone)}

8-74-1-2, $63 \mathrm{~cm} ; 2 \mathrm{~m}$ :

[All coccoliths rare] Ceratolithus cristatus, Coccolithus pelagicus, Cyclococcolithina leptoporus, Gephyrocapsa oceanica. Reworked taxa: Cyclococcolithina macintyrei, Discoaster deflandrei, Reticulofenestra umbilica.

\section{Lower Miocene}

(Triquetrorhabdulus carinatus Zone)

8-74-3-4, $63 \mathrm{~cm} ; 21 \mathrm{~m}$ :

Coccolithus sp. aff. C. bisectus, C. eopelagicus, Cyclococcolithina neogammation, D. deflandrei, Sphenolithus moriformis, Triquetrorhabdulus carinatus.

8-74-4-4, $63 \mathrm{~cm} ; 31 \mathrm{~m}:$

C. sp. aff. C. bisectus, C. eopelagicus, $C$. pelagicus. $C$. neogammation, $D$. deflandrei, Helicopontosphaera parallela [rare], Sphenolithus sp. aff. S. belemnos, $S$. moriformis, $T$. carinatus.

8-74-5-2, $63 \mathrm{~cm} ; 38 \mathrm{~m}$ :

C. eopelagicus, $C$. pelagicus, C. neogammation, Discoaster adamanteus, $D$. deflandrei, $D$. sp. cf. D. lidzi, T. carinatus [abundant] .

8-74-5-6, $63 \mathrm{~cm} ; 44 \mathrm{~m}$ :

$C$. eopelagicus, $C$. pelagicus, C. neogammation, $D$. deflandrei, $S$. sp. aff. S. belemnos, T. carinatus [abundant].

\section{Upper Oligocene \\ (Sphenolithus ciperoensis Zone)}

8-74-6-2, $63 \mathrm{~cm} ; 48 \mathrm{~m}$ :

Coccolithus bisectus, $C$. sp. aff. C. bisectus, C. eopelagicus, C. pelagicus, C. neogammation, D. deflandrei, $D$. sp. cf. $D$. lidzi, $S$. sp. aff. $S$. belemnos, $S$. ciperoensis, $S$. moriformis, $T$. carinatus. 
8-74-7-5, 63-64-cm; $61 \mathrm{~m}$ :

$C$. sp. aff. $C$. bisectus, $C$. eopelagicus, $C$. neogammation, $D$. deflandrei, $S$. sp. aff. $S$. belemnos, $S$. ciperoensis, $S$. moriformis, $T$. carinatus.

\section{Middle Oligocene \\ (Sphenolithus predistentus Zone)}

8-74-8-2, 63-64 cm; $66 \mathrm{~m}$ :

$C$. bisectus, $C$. eopelagicus, $C$. pelagicus, $C$. neogammation, D. deflandrei, D. tani nodifer, D. tani tani, Sphenolithus predistentus, S. pseudoradians [rare].

8-74-8-3, 63-64 cm; $68 \mathrm{~m}$ :

C. eopelagicus, $C$. pelagicus, $C$. neogammation, $D$. deflandrei, D. tani nodifer, D. tani tani, Sphenolithus distentus, $S$. predistentus, $S$. moriformis, $S$. pseudoradians.

8-74-9-5, 63-64 cm; $79 \mathrm{~m}$ :

$C$. bisectus, $C$. pelagicus, $C$. neogammation, $D$. deflandrei, D. tani nodifer, D. tani tani, Helicopontosphaera compacta, S. moriformis, $S$. predistentus.

8-74-10-1, 19-20 cm; $82 \mathrm{~m}$ :

C. bisectus, C. eopelagicus, C. pelagicus, Coccolithus stavensis, $C$. neogammation, $D$. deflandrei, $D$. sp. $\mathrm{cf} . D$. lidzi, D. tani tani, Pontosphaera vadosa, Reticulofenestra gartneri, $S$. distentus, $S$. predistentus, $S$. moriformis.

\section{Lower Oligocene \\ (Helicopontosphaera reticulata Zone)}

8-74-11-1, $63 \mathrm{~cm} ; 91 \mathrm{~m}$ :

Bramletteius serraculoides, $C$. bisectus, $C$. eopelagicus, $C$. pelagicus, $C$. stavensis, $C$. formosus, $C$. neogammation, D. deflandrei, D. tani nodifer, D. tani tani, $H$. compacta, Isthmolithus recurvus [rare], $P$. vadosa, Reticulofenestra umbilica, $S$. distentus, $S$. predistentus, S. moriformis.

\section{Middle Eocene \\ (Reticulofenestra umbilica Zone)}

8-74-12-3, 24-25 cm; $102 \mathrm{~m}$ :

Campylosphaera dela, Chiasmolithus grandis, Coccolithus eopelagicus, $C$. formosus, Discoaster barbadiensis, D. wemmelensis, Helicopontosphaera sp. cf. $H$. compacta, Sphenolithus furcatolithoides.

\section{HOLE 75}

(lat $12^{\circ} 31.00^{\prime} \mathrm{S}$., long $134^{\circ} 16.00^{\prime} \mathrm{W}$., depth 4181 meters)

\section{Summary of Coccolith Stratigraphy}

Lower Miocene sediment of the Discoaster druggii Subzone is present in Core 1 at 4 meters below the sea floor. The lowest Miocene sample occurs in Core 4 at about 31 meters. Below, upper and middle Oligocene assemblages are present to the deepest sample available in Core 9 at 80 meters. The very shallow occurrence of lower Miocene strata at this site is the culmination of a trend in which the base of the Miocene occurs at progressively shallower depth southward from Site 71 (Figure 2). The Oligocene-Miocene boundary is at approximately 370 meters at Site 71 (latitude $4^{\circ}$ $28.28^{\prime}$ N.), 270 meters at Site 72 (latitude $0^{\circ} 26.49^{\prime}$ N.), 148 meters at Site 73 (latitude $1^{\circ} 54.58^{\prime} \mathrm{S}$.), 64 meters at Site 74 (latitude $6^{\circ} 14.20^{\prime} \mathrm{S}$.), and 32 meters at Site 75 (latitude $12^{\circ} 31.00^{\prime} \mathrm{S}$.). This relationship is apparently a joint result of diminishing sedimentation rate and, as at Site 73 , of unconformity within the middle to upper Miocene section south of the equatorial zone.

\section{Coccoliths in Selected Samples, Hole 75}

\section{Lower Miocene \\ (Triquetrorhabdulus carinatus Zone, Discoaster druggii Subzone)}

8-75-1-4, $63 \mathrm{~cm} ; 4 \mathrm{~m}$ :

Coccolithus eopelagicus, C. pelagicus, Cyclococcolithina neogammation, Discoaster deflandrei, D. druggii, D. sp. cf. D. exilis, Orthorhabdus serratus, Sphenolithus sp. aff. $S$. belemnos, Triquetrorhabdulus carinatus.

\section{Lower Miocene \\ (Triquetrorhabdulus carinatus Zone, Coccolithus sp. aff. C. bisectus Subzone)}

8-75-2-5, $63 \mathrm{~cm} ; 16 \mathrm{~m}$ :

Coccolithus sp. aff. C. bisectus, C. eopelagicus, C. neogammation, D. deflandrei, Sphenolithus belemnos, $S$. sp. aff. $S$. belemnos, $T$. carinatus.

8-75-4-2, $63-64 \mathrm{~cm} ; 29 \mathrm{~m}$ :

$C$. sp. aff. C. bisectus, C. pelagicus, C. neogammation, $D$. deflandrei, $S$. sp. aff. S. belemnos, S. moriformis, $T$. carinatus.

\section{Upper Oligocene \\ (Sphenolithus ciperoensis Zone)}

8-75-4-5, 63-64 cm; $34 \mathrm{~m}$ :

$C$. sp. aff. C. bisectus, C. eopelagicus, $C$. pelagicus, $C$. neogammation, $D$. deflandrei, $S$. sp. aff. $S$. belemnos, $S$. ciperoensis, $S$. moriformis, $T$. carinatus.

8-75-5-5, $63-64 \mathrm{~cm} ; 43 \mathrm{~m}$ :

C. sp. aff. C. bisectus, C. eopelagicus, C. pelagicus, $C$. neogammation, $D$. deflandrei, $S$. ciperoensis, $S$. distentus, $S$. moriformis, $T$. carinatus. 


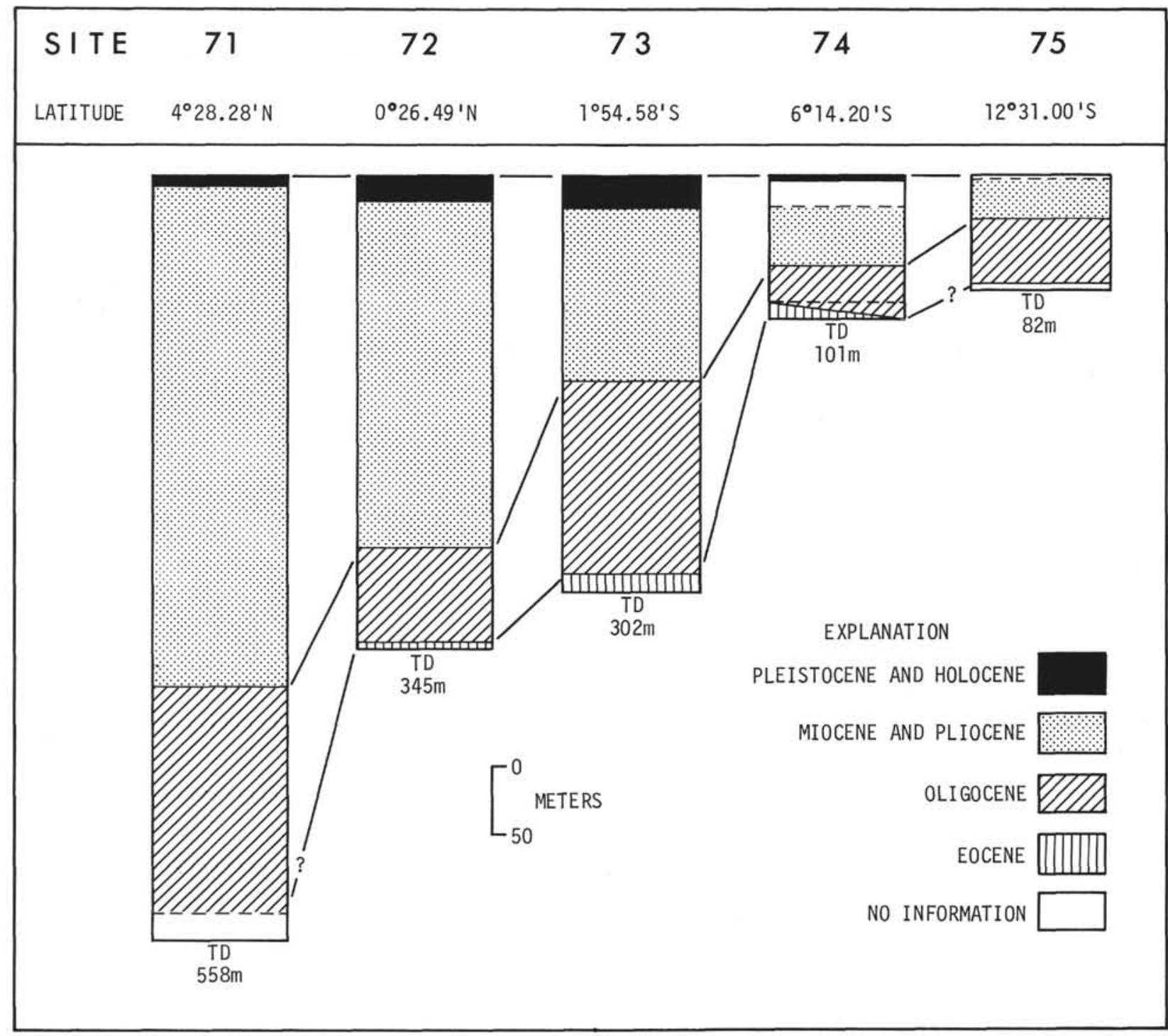

Figure 2. Comparative thickness of Cenozoic series along a south-trending transect in the central Pacific as indicated by the coccolith stratigraphy of cores from Leg 8, Deep Sea Drilling Project.

\section{Middle Oligocene}

(Sphenolithus predistentus Zone)

8-75-8-5, 63-64 cm; $71 \mathrm{~m}$ :

Coccolithus bisectus, $C$. sp. aff. C. bisectus, C. eopelagicus, C. neogammation, D. deflandrei, D. tani tani, Helicopontosphaera compacta, $S$. distentus, $S$. predistentus, $S$. pseudoradians.

8-75-9-2, $63-64 \mathrm{~cm} ; 75 \mathrm{~m}$ :

C. bisectus, $C$. sp. aff. C. bisectus, $C$. eopelagicus, $C$. neogammation, $D$. deflandrei, $D$. sp. cf. D. tani nodifer, $D$. tani tani, $S$. distentus, $S$. predistentus, $S$. pseudoradians.

\section{8-75-9-5, 63-64 cm; $80 \mathrm{~m}$ :}

$C$. bisectus, $C$. sp. aff. C. bisectus, $C$. eopelagicus, $C$. pelagicus, Coccolithus stavensis, $D$. deflandrei, D. tani nodifer, D. tani tani, Pontosphaera vadosa, $S$. distentus [rare], S. moriformis, S. predistentus.

\section{REFERENCES}

Bramlette, M. N., 1957. Discoaster and some related microfossils. U. S. Geol. Surv. Prof. Paper. 280-F, 247.

Bramlette, M. N., 1960. Age relations in early Tertiary of Europe and America as indicated by coccolithophorids and related microfossils. Bull. Geol. Soc. Am. 71, 1832 . 
Bramlette, M. N. and Bukry, D., 1968. Ranges of Tertiary nannoplankton. JOIDES Deep Sea Drilling Project, Core description manual. pt. 7, sec. 2, table $3 a$.

Bramlette, M. N. and Riedel, W. R., 1954. Stratigraphic value of discoasters and some other microfossils related to recent coccolithophores. J. Paleontology. 28,403 .

Bramlette, M. N. and Wilcoxon, J. A., 1967. Middle Tertiary calcareous nannoplankton of the Cipero Section, Trinidad W. I. Tulane Studies Geology. 5, 93.

Bukry, D., 1971. Coccolith stratigraphy Leg 7, Deep Sea Drilling Project. In Winterer, E. L. et al., 1971. Initial Reports of the Deep Sea Drilling Project, Volume VII. Washington (U. S. Government Printing Office).

Bukry, D. and Bramlette, M. N., 1968. Stratigraphic significance of two genera of Tertiary calcareous nannofossils. Tulane Studies Geology. 6, 149.

Bukry, D. and Bramlette, M. N., 1969. Some new and stratigraphically useful calcareous nannofossils of the Cenozoic. Tulane Studies Geology, Paleontology. 7, 131.
Bukry, D. and Bramlette, M. N., 1970. Coccolith age determinations Leg 3, Deep Sea Drilling Project. In Maxwell, A. E., et al., 1970. Initial Reports of the Deep Sea Drilling Project, Volume III. Washington (U. S. Government Printing Office).

Gartner, S., Jr., 1969. Correlation of Neogene planktonic foraminifer and calcareous nannofossil zones. Gulf Coast Assoc. Geol. Soc. Trans. 19, 585.

Hay, W. W., 1964. Utilisation stratigraphique des Discoasterides pour la zonation du Paleocene et de l'Eocene inferieur. Bureau Rech. Geol. et Min. Mem. 28,885 .

Hay, W. W., Mohler, H., Roth, P. H., Schmidt, R. R. and Boudreaux, J. E., 1967. Calcareous nannoplankton zonation of the Cenozoic of the Gulf Coast and Caribbean-Antillean area, and transoceanic correlation. Gulf Coast Assoc. Geol. Soc. Trans. 17, 428.

Martini, E. and Bramlette, M. N., 1963. Calcareous nannoplankton from the experimental Mohole drilling. J. Paleontology. 37, 845 . 